\title{
Modulation of neural oscillations during working memory update, maintenance, and readout: An hdEEG study
}

\author{
Marianna Semprini $^{1}$ (1) | Gaia Bonassi ${ }^{2}$ | Federico Barban ${ }^{1,3}$ | Elisa Pelosin ${ }^{4,5}$ |

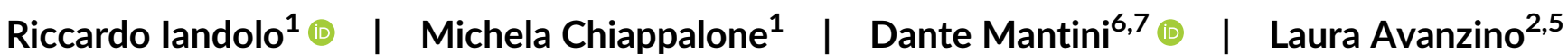

\footnotetext{
${ }^{1}$ Rehab Technologies, Istituto Italiano di Tecnologia, Genoa, Italy

${ }^{2}$ Department of Experimental Medicine, Section of Human Physiology, University of Genoa, Genoa, Italy

${ }^{3}$ Department of Informatics, Bioengineering, Robotics and System Engineering, University of Genoa, Genoa, Italy

${ }^{4}$ Department of Neuroscience, Rehabilitation, Ophthalmology, Genetics, Maternal and Child Health, University of Genoa, Genoa, Italy

${ }^{5}$ Ospedale Policlinico San Martino, IRCCS,

Genoa, Italy

${ }^{6}$ Research Center for Motor Control and Neuroplasticity, KU Leuven, Leuven, Belgium

${ }^{7}$ Brain Imaging and Neural Dynamics Research Group, IRCCS San Camillo Hospital, Venice, Italy
}

Correspondence

Laura Avanzino, Department of Experimental Medicine, Section of Human Physiology, University of Genoa, Genoa, Italy.

Email: lavanzino76@gmail.com

Funding information

Jacques und Gloria Gossweiler-Stiftung

\begin{abstract}
Working memory (WM) performance is very often measured using the n-back task, in which the participant is presented with a sequence of stimuli, and required to indicate whether the current stimulus matches the one presented $\mathrm{n}$ steps earlier. In this study, we used high-density electroencephalography (hdEEG) coupled to source localization to obtain information on spatial distribution and temporal dynamics of neural oscillations associated with WM update, maintenance and readout. Specifically, we a priori selected regions from a large fronto-parietal network, including also the insula and the cerebellum, and we analyzed modulation of neural oscillations by event-related desynchronization and synchronization (ERD/ERS). During update and readout, we found larger $\theta$ ERS and smaller $\beta$ ERS respect to maintenance in all the selected areas. $\gamma_{\text {LOW }}$ and $\gamma_{\text {HIGH }}$ bands oscillations decreased in the frontal and insular cortices of the left hemisphere. In the maintenance phase we observed decreased $\theta$ oscillations and increased $\beta$ oscillations (ERS) in most of the selected posterior areas and focally increased oscillations in $\gamma_{\text {LOW }}$ and $\gamma_{\text {HIGH }}$ bands in the frontal and insular cortices of the left hemisphere. Finally, during WM readout, we also found a focal modulation of the $\gamma_{\text {Low }}$ band in the left fusiform cortex and cerebellum, depending on the response trial type (true positive vs. true negative). Overall, our study demonstrated specific spectral signatures associated with updating of memory information, WM maintenance, and readout, with relatively high spatial resolution.

KEYWORDS

ERS/ERD, hdEEG, n-back, network, neural oscillations, working memory
\end{abstract}

\section{1 | INTRODUCTION}

The n-back task-first described by Kirchner in 1958 (Kirchner, 1958)-is the most popular task used to measure working memory (WM), relying on the presentation of "rapidly, continuously

Marianna Semprini and Gaia Bonassi contributed equally to this work. changing information" to measure very short-term retention. In this task, participants are presented with a series of stimuli and are asked to indicate whether the current stimulus (probe) matches the stimulus presented $n$-stimuli back in the series. A recent review highlighted that WM at $n$-back is associated with a cerebral network that varies with stimulus type, presentation modalities and as a function of processing load (Mencarelli et al., 2019). Additionally, a number of

This is an open access article under the terms of the Creative Commons Attribution License, which permits use, distribution and reproduction in any medium, 
evidence showed that specific frequency bands of electroencephalography (EEG) oscillations are of particular relevance for aspects of WM, such as the positive association between $\gamma$ band activity $(>40 \mathrm{~Hz})$ and performance at higher WM loads in healthy populations (Crone, Sinai, \& Korzeniewska, 2006; Honkanen, Rouhinen, Wang, Palva, \& Palva, 2015; Kucewicz et al., 2017; Lachaux, Axmacher, Mormann, Halgren, \& Crone, 2012; Roux, Wibral, Mohr, Singer, \& Uhlhaas, 2012) and the association between $\theta$ oscillations and WM (Brookes et al., 2011; Burke et al., 2013; Hsieh \& Ranganath, 2014). These observations have recently led to the use of noninvasive brain stimulation in combination with cognitive training for improving WM function (Hill, Rogasch, Fitzgerald, \& Hoy, 2019; Hoy et al., 2015; Jones, Johnson, \& Berryhill, 2020; Reinhart \& Nguyen, 2019). Particularly, transcranial Alternating Current Stimulation (tACS) (Antal \& Paulus, 2013; Helfrich et al., 2014) in the EEG range (conventionally: $0.1-80 \mathrm{~Hz}$ ) in the frontal cortex is believed to directly modulate cortical oscillations and to impact sensory, perceptual and cognitive processes (Herrmann, Rach, Neuling, \& Struber, 2013). However, to optimize such neuromodulation approach in cognitive rehabilitation of WM, we need a clear picture of the spatial distribution and temporal dynamics of cortical oscillations in the cerebral network involved in WM. In this context, high-density electroencephalography (hdEEG) provides us the possibility to gain information on the sources of the electrical oscillations underpinning cognitive processing with an optimal temporal resolution and an improved spatial resolution with respect to standard EEG (Michel et al., 2012). In particular, it is also fundamental to separately analyze oscillatory activity in the different phases of WM process: from the early phase of updating, that is, the stored information at stimulus presentation, up to the usage of such information to guide action, going through the maintenance of information in face of other stimuli. Albeit it is not easy to disentangle the classic phases of working memory process (update, maintenance, readout) in the $n$-back task, in this study we aim to obtain information on spatial location and temporal dynamics of neural activity associated with the different phases. To this end, we used a custom developed pipeline for performing source localization from hdEEG data. This pipeline is able to detect multiple brain networks that are spatially similar to those obtained from fMRI data (Liu, Farahibozorg, Porcaro, Wenderoth, \& Mantini, 2017; Liu, Ganzetti, Wenderoth, \& Mantini, 2018; Zhao, Marino, Samogin, Swinnen, \& Mantini, 2019).

We focused on correct update of stimuli by analyzing activity that was followed by a correct press $\mathrm{n}$ letters after (true positive) and activity that was followed by a correct no-press $\mathrm{n}$ letters after (true negative), with $\mathrm{n}$ being either 2 (2-back task) or 3 (3-back task). Furthermore, we analyzed hdEEG activity during the maintenance and readout of the $n$-back task. To analyze the maintenance phase, we observed the hdEEG activity in the single (2-back task) or in the two (3-back task) presented letters preceding the probe. Correct maintenance was identified when a correct response followed the appearance of the probe on the screen and when a correct noresponse followed the appearance of the probe on the screen. Finally, hdEEG activity during the presentation of the probe was used to analyze the readout identified by probe letters correctly recognized as matching or nonmatching the stimulus letter presented ntrials earlier.

We here performed analysis of source reconstructed data filtered in different frequency bands and demonstrated specific spectral signatures associated with updating of memory information, WM maintenance and readout.

\section{2 | MATERIALS AND METHODS}

\subsection{Data collection}

We recruited 21 neurologically intact, right-handed subjects (9 females, age $30.9 \pm 6.8$ years, mean $\pm S D$ ). All subjects provided written informed consent. The study conforms to the standard of the Declaration of Helsinki and was approved by the institutional ethical committee (CER Liguria Ref.1293 of September 12, 2018).

The behavioral task consisted in a n-back working memory (WM) task (with $n=2,3$ ) as in (Hoy et al., 2015). Briefly, a series of random letters (A, B, C, D, E, F, G, H, I, O) was visually presented in sequence and the subject was required to respond with a button press when the currently presented letter corresponded to the letter presented $\mathrm{n}$ trials earlier (Figure 1a). Each letter appeared on a screen for $500 \mathrm{~ms}$ with a $2000 \mathrm{~ms}$ delay between stimuli presentations (Figure 1c). Each subject performed the task twice, first with $n=2$ than with $n=3$, or vice versa. The order of task execution was randomly assigned to the subjects.

For hdEEG recording we used a 128 channel EEG recording system (actiCHamp, Brain Products) with electrodes arranged according to the 5-10 system (Oostenveld \& Praamstra, 2001) and equipped with a trigger box handling external events. We collected hdEEG data at $1000 \mathrm{~Hz}$ sampling frequency, using the electrode $\mathrm{FCz}$ as physical reference. We also collected horizontal and vertical electrooculograms (EOG) from the right eye for further identification and removal of ocular-related artifacts.

The behavioral task was handled by a custom graphical user interface (GUI) developed in Matlab (The MathWorks). The GUI ran on a dedicated computer and was also responsible for sending task-related triggers to the EEG recording system. These triggers were sent through a NI USB board (National Instruments), which was also responsible of informing both the EEG recording system and the pc used for the cognitive task of a button press event.

\subsection{Cognitive processes underpinning working memory}

With respect to the cognitive processes involved during the task, we distinguished between three WM phases: update, maintenance, and readout. According to this distinction, the presented letters assume 
(a)

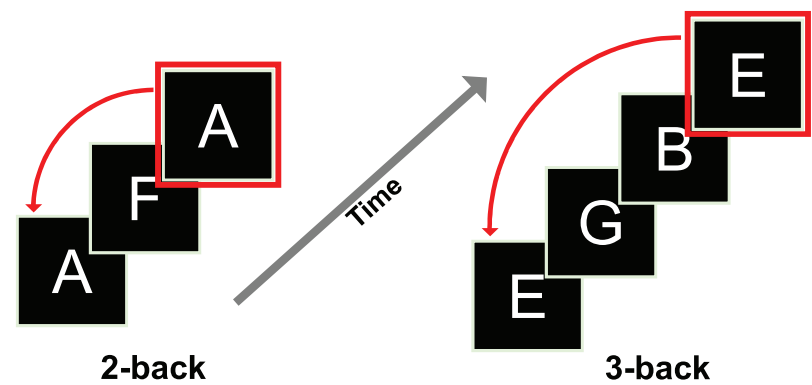

(b)

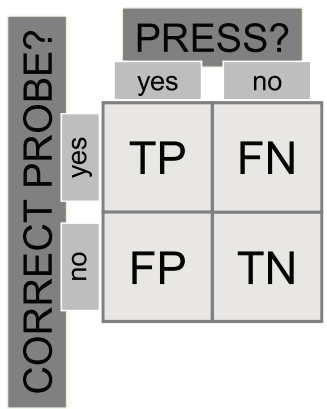

(c)
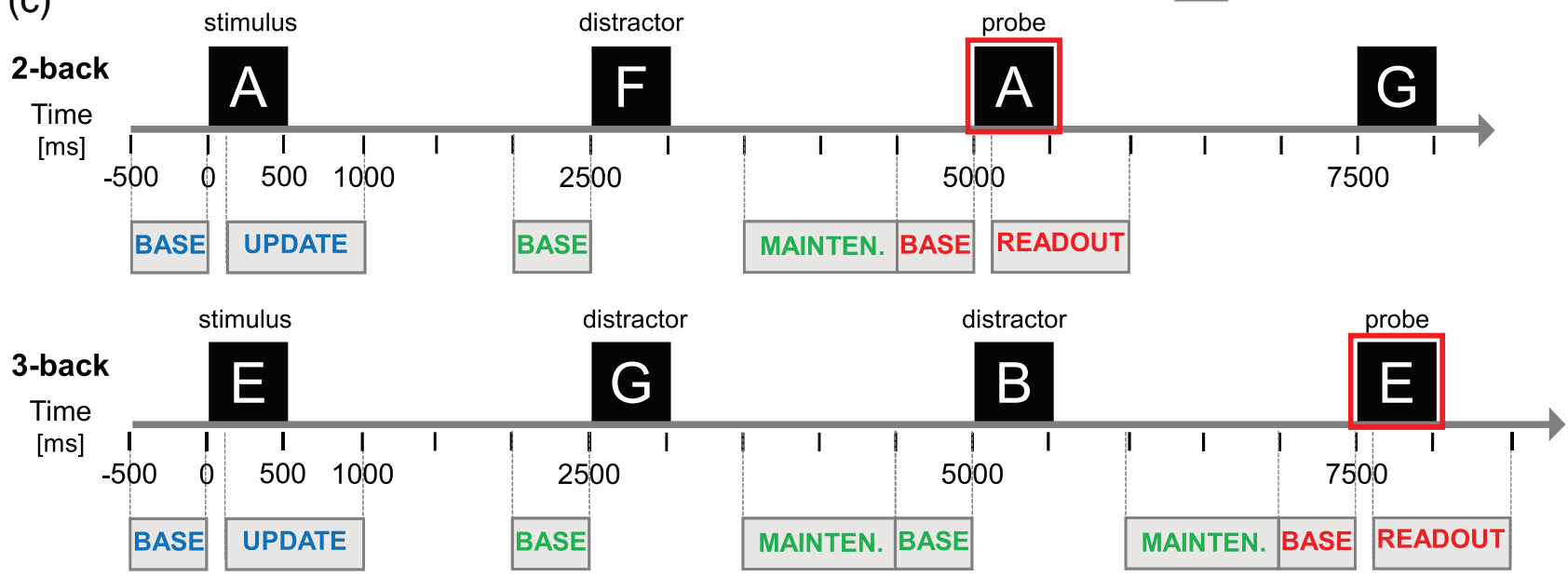

FIGURE 1 Outline of working memory task. (a) Graphical representation of n-back tasks: the current letter (framed in red) must be compared with the one presented $n$ times before, $n$ being either 2 or 3 depending on the task. (b) Contingency matrix of possible behavioral outcomes: true positive (TP), true negative (TN), false positive (FP), and false negative (FN). (c) Timeline representing 2-back (top) and 3-back (bottom) task timings and intervals chosen for hdEEG analysis. Letters appear on screen every $2500 \mathrm{~ms}$ and remain displayed for 500 ms. Analysis of memory update was performed by comparing baseline (500 ms preceding letter presentation, indicated as "BASE" in the figure) with a portion of signal ranging from 100 to $1000 \mathrm{~ms}$ post stimulus-letter onset (900 ms in total). Analysis of memory update was performed by comparing baseline (500 ms preceding distractor-letter presentation) with a portion of signal ranging from 1000 to 2000 ms post distractor-letter onset (1000 ms in total). Analysis of readout was performed by comparing baseline (500 ms preceding probe-letter presentation) with a portion of signal ranging from 100 to $600 \mathrm{~ms}$ post probe-letter onset (500 ms in total)

different roles. As an example, in Figure 1a, for the 2-back case, the framed " $A$ " represents the probe letter, the other " $A$ " is the stimulus letter, and the " $F$ " is the distractor; For the 3-back case, the framed " $E$ " represents the probe letter, the other " $E$ " is the stimulus letter, and " $G$ " and "B" are distractors. During the task, each letter was simultaneously a stimulus (with respect to the letter appearing $n$ trials later), a distractor (with respect to the letter appearing $n-1$ [and $n-2$, for $n=3$ ] trial later), and a probe (with respect to the letter that was presented $\mathrm{n}$ trials before).

Memory update refers to the process of storing the presented letter (stimulus) for future comparison with the next probe letter (probe being either 2 or 3 trials later, depending on $n$ value).

Memory maintenance refers to the process of keeping the previously presented letter in memory, when other letters (distractors) are presented before the probe letter (there is one distractor letter in the 2-back case, and two distractor letters in the 3-back case).

Readout corresponds to the processing of a behavioral response after the probe letter has been presented.
As depicted in Figure 1b, by observing the behavioral responses we distinguished trials as belonging to one of the following categories:

- True positive (TP): probe letter correctly recognized as matching the stimulus letter (button press).

- True negative (TN): probe letter correctly recognized as nonmatching the stimulus letter (no button press).

- False positive (FP): probe letter incorrectly recognized as matching the stimulus letter (button press).

- False negative (FN): probe letter incorrectly recognized as nonmatching the stimulus letter (no button press).

In this work, we only observed brain responses during the wellperformed trials, that is, TP and TN, because the number of the badly performed trials (FP and FN) was too small. Indeed, the 2- and 3-back tasks, although challenging, are generally performed by healthy subjects with high levels of success. We indeed found that our subjects 
reached high levels of accuracy in both tasks, and the small number of incorrect trials made impossible to statistically validate the analysis of their related neural activity.

We observed task performance by computing reaction time, defined as the delay between probe letter onset and button press for TP trials only, and accuracy, defined as the ratio of TP trials over the total number of response (TP + TN + FP + FN).

\section{3 | hdEEG preprocessing and source localization}

For analysis of hdEEG data, we made use of a tailored analysis pipeline that was recently developed to reconstruct source of neural oscillations from cortical/subcortical gray matter and cerebellar gray matter (Liu et al., 2017).

We first attenuated the power noise in the EEG channels by using a notch filter centered at $50 \mathrm{~Hz}$. Then, we detected channels with low signal to noise ratio and we labeled them as "bad channels." We defined a channel as "bad" if it resulted as an outlier with respect to: (a) the Pearson correlation of the signal in the frequency band 1-80 Hz against all the signals from all the other channels; and/or (b) the noise variance estimated in the frequency band $200-250 \mathrm{~Hz}$, where the EEG contribution can be considered as negligible. The threshold to define an outlier was set to mean $\pm 3 S D$ of the values. The bad channels were interpolated by using information coming from the neighboring channels, as implemented in the FieldTrip toolbox (http://www.fieldtriptoolbox.org/). EEG signals were then band-pass filtered $(1-80 \mathrm{~Hz})$ with a FIR zero-phase distortion filter and downsampled at $250 \mathrm{~Hz}$.

Biological artifacts were rejected using Independent Component Analysis (ICA). Independent Components (ICs) were estimated with a fast fixed-point ICA (FastICA) algorithm (Hyvarinen \& Oja, 2000), as described in (Mantini, Franciotti, Romani, \& Pizzella, 2008). ICs were marked as bad if correlation with the time course of the EOG signals was higher than 0.2. The time courses of the ICs classified as bad were reconstructed at the channel level and subtracted from the data. EEG signals were then re-referenced with a customized version of the Reference Electrode Standardization Technique (REST) (Liu et al., 2015; Mantini et al., 2008; Yao, 2001; Yao et al., 2005).

As in (Liu et al., 2017), we generated a volume conductor head model using a 128 electrodes template positioned over a T1-weighted MR anatomical template. Then, we segmented 12 tissue classes: skin, eyes, muscle, fat, spongy bone, compact bone, gray matter, cerebellar gray matter, white matter, cerebellar white matter, cerebrospinal fluid and brainstem and we assigned them with characteristics conductivity values, as in (Haueisen, Bottner, Funke, Brauer, \& Nowak, 1997). To create a numerical approximation of the volume conduction model and to calculate the leadfield matrix, we used the Simbio finite element method (FEM) implemented in FieldTrip. The leadfield matrix estimated the relationship between the measured scalp potentials and the dipoles corresponding to brain sources, which were constrained by a regular $6 \mathrm{~mm}$ grid spanning the cortical, subcortical, and cerebellar gray matter.
Sources reconstruction was performed with the exact lowresolution brain electromagnetic tomography eLORETA (PascualMarqui et al., 2011) algorithm, using both the artifacts-free hdEEG signals and the head model conductor.

\section{4 | ERS-ERD analysis}

We chose to analyze a specific set of regions of interest (ROIs) in the brain, whose activation was previously found related to the n-back task (Mencarelli et al., 2019). Table 1 summarizes the observed ROIs.

We computed event related synchronization and desynchr onization (ERS/ERD) of source reconstructed data filtered in different frequency bands and during different WM processing phases. Specifically, for each WM phase (update, maintenance and readout) we generated a spectrogram using Short-Time Fourier Transform for the frequency range $1-80 \mathrm{~Hz}$, at steps of $1 \mathrm{~Hz}$, and with temporal resolution equal to $100 \mathrm{~ms}$. The spectrogram was epoched, according to each specific condition (see below) and then averaged. Finally, we calculated ERD/ERS intensity as the power change of the signal in a specific time range with respect to a reference period (baseline) (Pfurtscheller, 2001). We chose as baseline the 500 ms preceding letter presentation in all cases, as in (Hoy et al., 2016).

The observed frequency bands were $\theta(4-8 \mathrm{~Hz}), \alpha(8-13 \mathrm{~Hz}), \beta$ $(13-30 \mathrm{~Hz}), \gamma_{\text {LOW }}(30-50 \mathrm{~Hz})$, and $\gamma_{\mathrm{HIGH}}(50-80 \mathrm{~Hz})$. The $\delta$ band (1-4 Hz) was excluded from analysis, because it is often contaminated by motion artifacts.

Time range for update was set between 100 and 1,000 ms post stimulus onset, similarly to (Hoy et al., 2016). The lower limit was set to $100 \mathrm{~ms}$ instead of $0 \mathrm{~ms}$, because the visual system takes up to $150 \mathrm{~ms}$ to process visual stimuli (Thorpe, Fize, \& Marlot, 1996).

Time range for maintenance was set between 1,000 and $2000 \mathrm{~ms}$ post distractor onset. We chose this interval in order to discount the contribution provided by the update $(100-1,000 \mathrm{~ms}$ post letter presentation) of the distractors, which are, at the same time, probe letters for the following trials.

Time range for readout was set between 100 and 600 ms poststimulus onset. The lower limit was chosen as for the other trials in order to take into account the processing delays of the visual system (Thorpe et al., 1996), while for the upper limit the choice was datadriven and calculated according to the press distribution of all subjects during TP trials. Briefly, we grouped the press times of all subjects during all TP trials during 2-back (507 trials in total) and during 3-back (357 trials in total) and calculated the first percentile of each distribution (686 and $639 \mathrm{~ms}$, respectively). We thus chose $600 \mathrm{~ms}$ as upper limit for both cases (2- and 3-back) and rejected trials where the press was made within $600 \mathrm{~ms}$ following letter presentation (in total we rejected 1 trial for 2-back and 7 trials for 3-back). The chosen temporal parameters are summarized in Table 2.

For statistical analysis of the data, we first assessed data normality with the one-sample Kolmogorov-Smirnov test. Then, a three-way repeated-measure analysis of variance (ANOVA) was run to test the influence on the mean ERD/ERS intensity on TASK (2-back and 
TAB LE 1 List of observed ROIs, areas they belong, and corresponding MNI coordinates

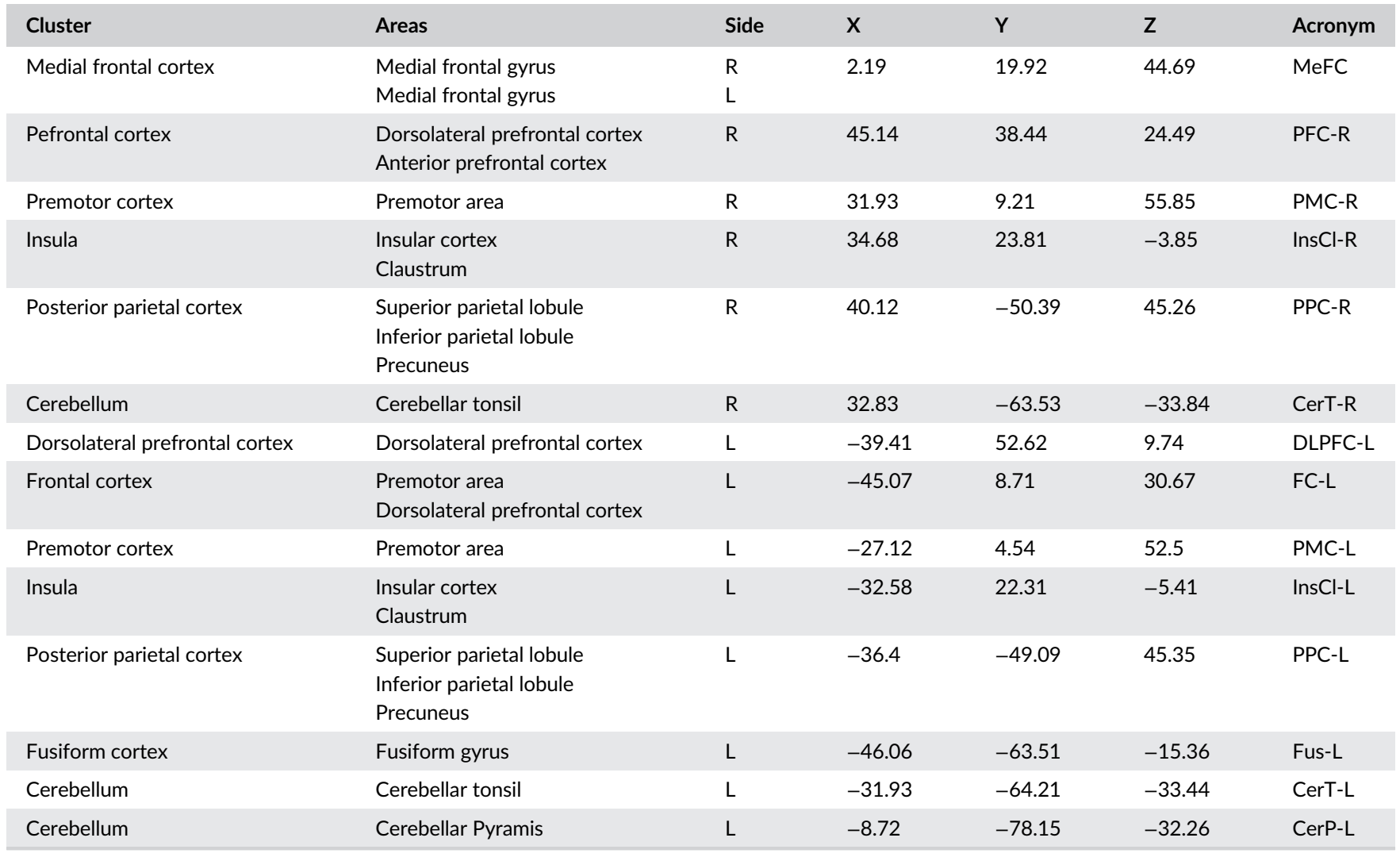

TABLE 2 Temporal parameters used for ERS/ERD analysis of WM processing

\begin{tabular}{|llll|}
\hline Condition & Reference $[0 \mathrm{~ms}]$ & Baseline & Time range $[\mathrm{ms}]$ \\
\hline Update & Target onset & {$[-500$ 0] } & {$[1001000]$} \\
\hline Maintenance, 2-back & Distractor onset & {$[-5000]$} & {$[1,000$ 2000] } \\
\hline Maintenance, 3-back & Distractor 1 onset & {$[-500$ 0] } & {$[1,0002000]$} \\
& Distractor 2 onset & {$[-5000]$} & {$[1,0002000]$} \\
\hline Readout & Probe onset & {$[-5000]$} & {$[100600]$} \\
\hline
\end{tabular}

3-back), PHASE (update, maintenance and readout) and TRIAL (TP, TN) as main factors within subjects, as well as of their interaction. This analysis was run separately for each ROI and for each frequency band. Post hoc analysis was performed with Fisher Least Significant Difference method. The significance level was set to 0.05 for all analyses.

\section{3 | RESULTS}

\section{1 | Working memory performance}

Due to a lower WM load, best performance was obtained for the 2-back than the 3-back task. Figure 2 reports single subjects' scores (panels $A$ and $D$ ) as well as average scores (panels $B$ and $E$ ) for the two tasks. We found a significant difference between accuracies obtained in the 2- and 3-back tasks (paired $t$ test, $p=.0042$ ) but not between reaction times (paired $t$ test, $p=.21$ ). In Figure $2 \mathrm{C}$ we report the normalized distributions of reaction times for the 2-back (top) and 3-back (bottom) task. In Figure $2 \mathrm{~F}$ we plotted single subjects' accuracy against mean reaction time for the two tasks. We found no correlation between these two measures.

\subsection{ERS/ERD analysis}

For a specific set of ROIs (Table 1), we computed ERS/ERD for different tasks (i.e., 2- and 3-back), different trial types (i.e., TP and TN) and during the different phases of the working memory task (i.e., update, maintenance, and readout).

We did not observe an effect of TASK on ERS/ERD modulation. Effect of TRIAL was observed in $\beta, \gamma_{\text {LOW }}$ and $\gamma_{\text {HIGH }}$ bands for few ROls, with TP showing ERS and TN showing ERD (see Supplementary Materials). For most of the ROls of interest, we observed a statistically significant effect of PHASE in all bands, except for $\alpha$. Significant interactions of main effects were never found between TASK and TRIAL, while in the other conditions (TASK*PHASE, TRIAL*PHASE and TASK*TRIAL*PHASE) were found for few ROIs in the $\gamma_{\text {LOW }}$ band. 
(a)
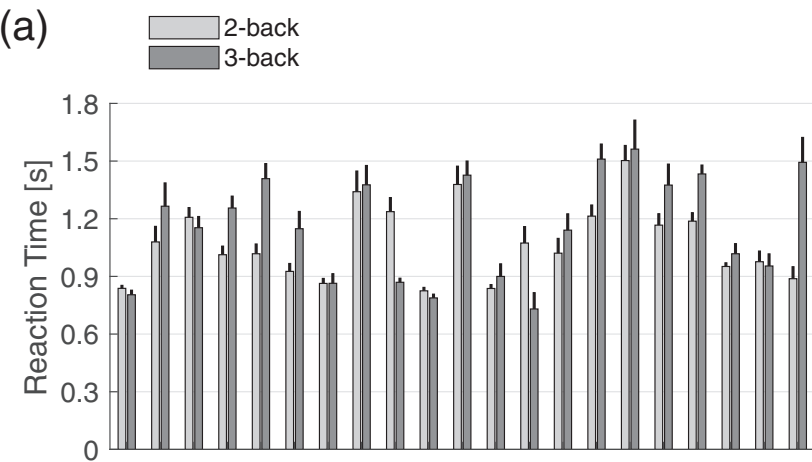

(d)

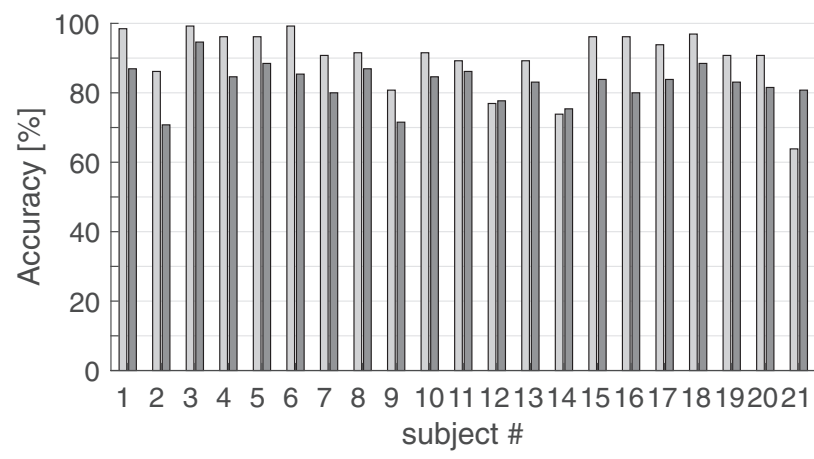

(b)

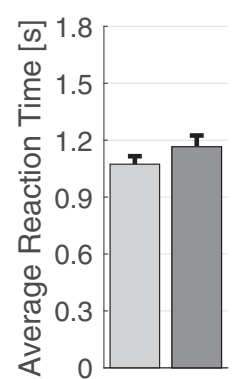

(e)

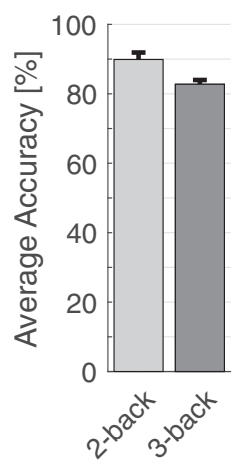

(c)
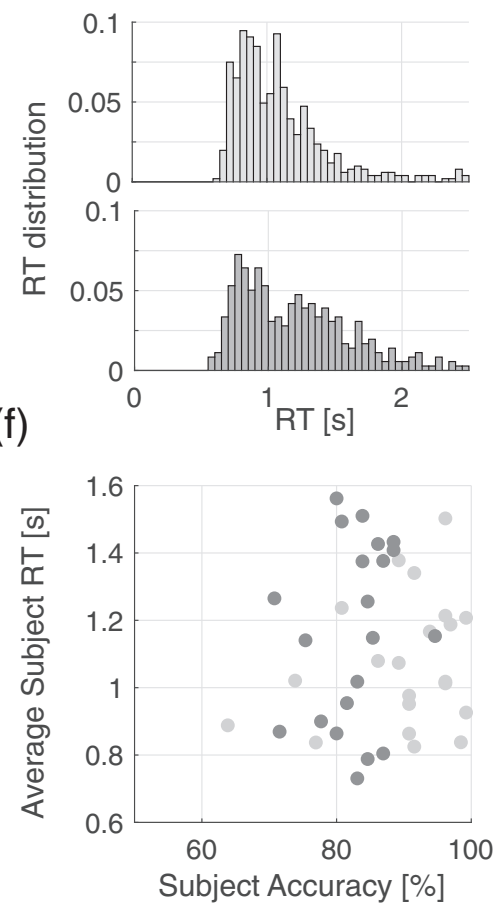

FIGURE 2 Cognitive performance of n-back task. (a) Reaction times obtained by single subjects during the 2-back (light gray) and 3-back (dark gray) task (mean \pm standard error of TP trials). (b) Average reaction time obtained in the two tasks (mean \pm standard error of single subjects' scores). (c) Normalized reaction times distribution of reaction times during the 2-back (top) and 3-back (bottom) tasks; data obtained from all the TP trials of all subjects. (d) Accuracy obtained by single subjects during the 2-back (light gray) and 3-back (dark gray) task. (e) Average reaction time obtained in the two tasks (mean \pm standard error of single subjects' scores). (f) Accuracy vs mean reaction times of single subjects in the 2-back (light gray dots) and 3-back (dark gray dots) task

\section{3 | Effect of PHASE}

In the WM network, during update and readout, we found larger $\theta$ oscillations and smaller $\beta$ oscillations respect to maintenance. In the maintenance phase we observed decreased $\theta$ oscillations with $\theta$ ERD in most of the selected posterior areas, and increased $\beta$ oscillations (ERS).

Figure 3 reports ERS/ERD modulation in $\theta / \beta$ bands for all the ROIs of interest. A significant effect of PHASE was observed for the $\theta$ band in all the ROls analyzed (Table $3 p \leq 10^{-5}$ in all cases; Figure 3 panel a). Post hoc analysis revealed an increase in $\theta$ oscillations in the update and readout with respect to maintenance, in all the analyzed ROIs $\left(p \leq 10^{-3}\right.$ in all cases, except DLPFC-L, FC-L, and CerP-L with $\leq 10^{-2}$; Figure 3). For the $\beta$ band, a significant effect of PHASE was also observed in all the ROls (Table $3 p \leq .001$ in all cases, except PFC-R with $p=.012$, Figure 3 panel a). Post hoc analysis revealed an increase in $\beta$ oscillation in the maintenance and a decrease in $\beta$ oscillation in the update and readout (maintenance vs. update and readout, $\mathrm{p} \leq 10^{-2}$ in all cases).

For $\gamma_{\text {LOW }}$ and $\gamma_{\text {HIGH }}$ bands, ERS/ERD modulation was focally modified in the left hemisphere, in the insular, frontal cortex and in the cerebellar ROls. Analogously to $\beta$ activity, we observed in the insular and frontal cortex, smaller oscillations in the update and the readout with respect to maintenance. ERS/ERD modulation in $\gamma_{\text {Low/ }}$ $\gamma_{\text {HIGH }}$ bands is reported in Figure 4 for the ROIs in which the effect of PHASE was found significant, and in Figure S1 for the other ROls. Indeed, a significant effect of PHASE was observed mostly in the left hemisphere (Figure 4 panel a): $\gamma_{\text {Low }}$ oscillations were found significant (Table 3, $p \leq .05$ ) in DLPFC-L, FC-L, IncCl-L, Fus-L, and CerT-L, while $\gamma_{\text {HIGH }}$ oscillations in CerT-R, DLPFC-L, IncCl-L, and CerTL. Post hoc analysis revealed a stronger increase in $\gamma$ oscillation (ERS) in the maintenance phase than in update and readout (maintenance vs. update and readout, $p \leq .04$, Figure 4).

\subsection{Interactions of main effects}

Interactions of main effects were found only in the $\gamma_{\text {Low }}$ band. TASK*PHASE interaction was observed in cerebellum, in both hemispheres (Figure 5, Table S2; CerT-R $p=.002$, CerP-L $p=.036$ ), with update during 3-back showing a significant stronger ERD than during 2-back in the CerT-R $(U$ (mean $\pm S D)$ : 2-back $(-0.97 \pm 1.13)$ 3-back $(-3.88 \pm 0.95)$, post hoc analysis, $p=.010)$ and a trend in the CerP-L $(U$ (mean $\pm S D):$-back $(-0.32 \pm 1.20)$ 3-back $(-2.84 \pm 0.82)$, post hoc analysis, $p=.09$ ).

In the left fusiform cortex and left cerebellum it was also observed a significant interaction TRIAL*PHASE (Figure 5, Table S3; 
(a)
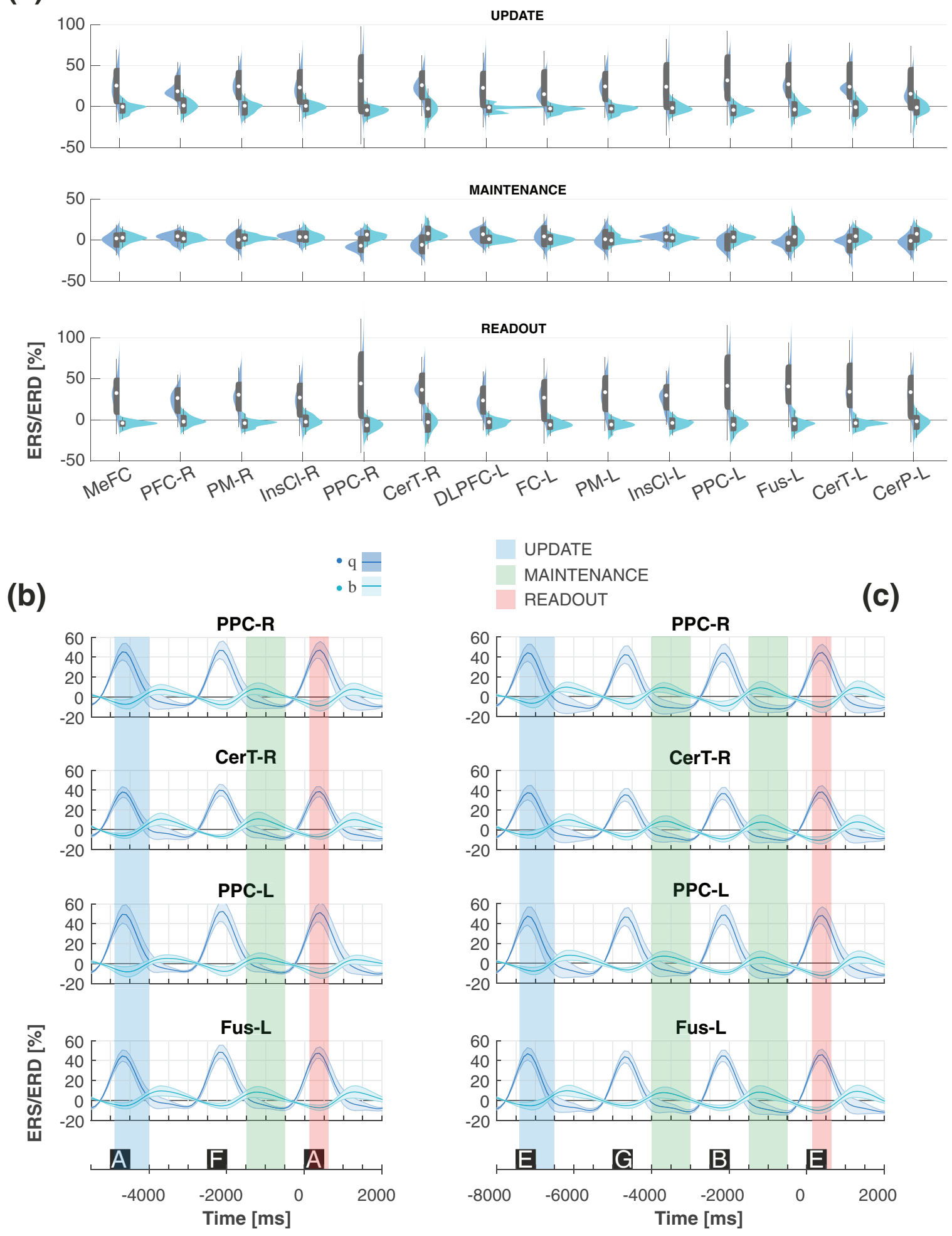

\section{UPDATE}

MAINTENANCE

READOUT

(c)

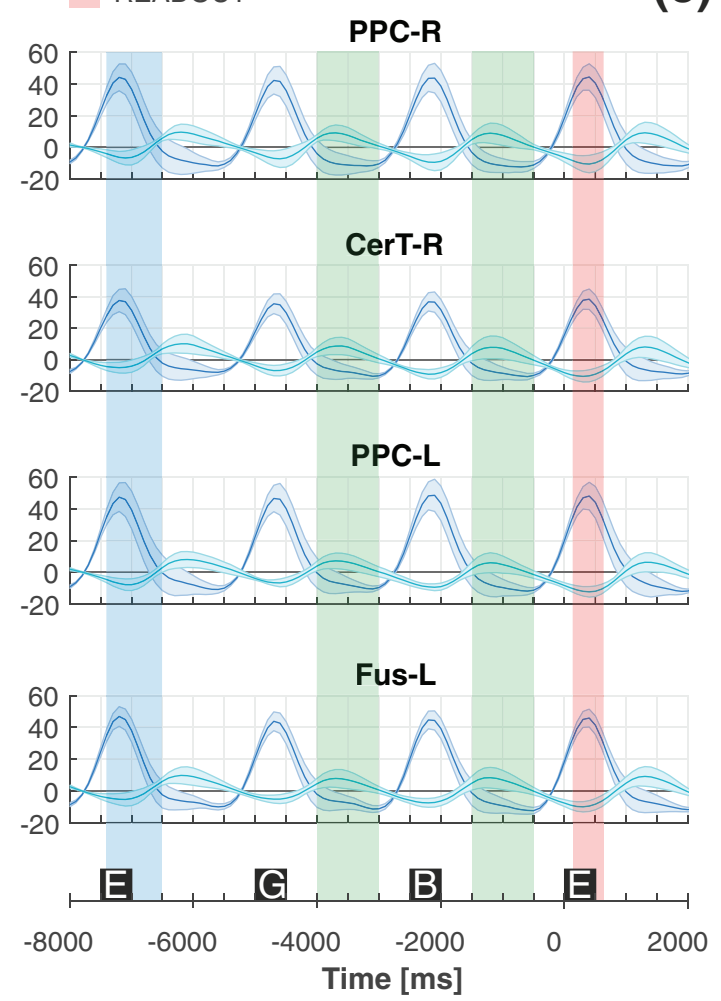

FIGURE 3 Effect of PHASE in the $\theta$ and $\beta$ band. (a) Violin plots of ERS/ERD variation in the $\theta$ (blue) and $\beta$ (light blue) bands during update (top), maintenance (middle) and readout (bottom). Superimposed in grey are boxplots describing the median value (white dot), 25th and 75th percentiles (extremes of the thick grey line), and full data range (extremes of the thin grey line) of the distributions. (b) Temporal evolution of band power in the $\theta$ (blue) and $\beta$ (light blue) during the 2-back task for PPC-R, CerT-R, PPC-L, and Fus-L). (c) Temporal evolution (mean - thick lines, standard deviation - shaded areas) of band power in the $\theta$ (blue) and $\beta$ (light blue) during the 3-back task for PPC-R, CerT-R, PPC-L, and Fus-L

CerT-L $p=.013$, Fus-L $p=.002$ ). Here we only expect interactions with the response phase, as trial type should not influence update and maintenance phases. Indeed, post hoc analysis was found significant for CerT-L (R (mean $\pm S D)$ : TP $(2.91 \pm 1.24)$ TN $(-2.44 \pm 0.82)$, 
TABLE 3 Results of ANOVA related to the main effect of PHASE

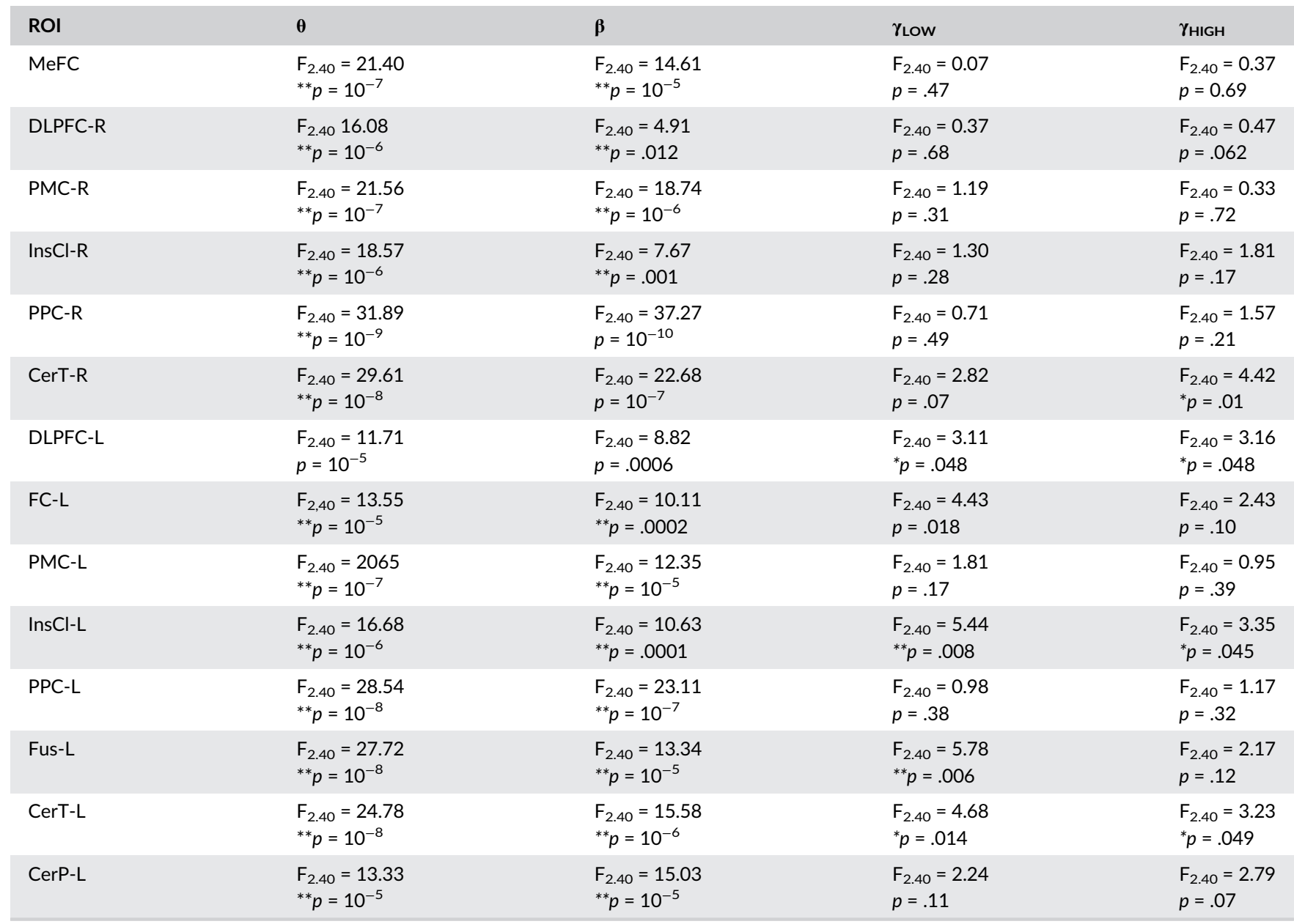

Note: Asterisks report the level of significance $\left({ }^{* *} p<.01{ }^{*} p<.05\right)$.

$p<.0005)$ and Fus-L $\quad(\mathrm{R} \quad($ mean $\pm S D): \quad$ TP $\quad(3.13 \pm 1.42) \quad$ TN $(-1.98 \pm 0.75), p=.0012)$, with $\gamma$ oscillations increasing during readout of TP trials and decreasing during readout of TN trials. Finally, a significant TASK*TRIAL*PHASE interaction was also observed in the left fusiform cortex and right cerebellum (Figure 5, Table S4; CerT-R $p=.017$, Fus $-\mathrm{L} p=.03$ ). Post hoc analysis showed, for TP trials in both areas, stronger ERD for the 3-back task with respect to the 2-back task in the update phase (TP $\cup($ mean $\pm S D)$ : 2-back $(0.71 \pm 2.07)$ 3-back $(-5.36 \pm 1.91), p=.006$ for CerT-R and TP $U$ (mean $\pm S D)$ : 2-back $(-0.29 \pm 1.81$ ) 3-back $(-8.5 \pm 2.32), p=.014$ for Fus-L). In CerT-R we also found a stronger ERS in the 3-back task with respect to the 2-back in the readout phase of TP trials $(p=.048)$.

\section{4 | DISCUSSION}

The main goal of this study was to obtain information on spatial location and temporal dynamics of neural oscillations associated with the different phases of working memory (update, maintenance, readout) in the n-back task.
Indeed, working memory is a high cognitive function that refers to the ability to encode, manipulate and retrieve information online and over a limited period of time (Baddeley, 1996). To this aim, taking advantage of recent developments on the accurate reconstruction of neural activity in the brain from hdEEG (Liu et al., 2017; Zhao et al., 2019), we analyzed spectral signatures associated with updating of memory information, its maintenance and its readout when used to inform and guide behavior. We a priori selected a large fronto-parietal network (Mencarelli et al., 2019), including also the insula, involved in memory storage, and the cerebellum as subcortical area that has demonstrated to be involved in cognitive functions (Strick, Dum, \& Fiez, 2009).

The main results of the present study were the following: (a) in the update and readout, larger $\theta$ oscillations accompanied by smaller $\beta$ oscillations in most of the selected areas and decreased $\gamma_{\text {Low }}$ and $\gamma_{\text {HIGH }}$ bands in the frontal and insular cortices of the left hemisphere; (b) in the maintenance, decreased $\theta$ oscillations and increased $\beta$ oscillation in posterior areas and increased oscillation in $\gamma_{\text {LOW }}$ and $\gamma_{\text {HIGH }}$ bands in the frontal and insular cortices of the left hemisphere; (c) in the readout, focal modulation of $\gamma_{\text {Low }}$ band in the 
(a)
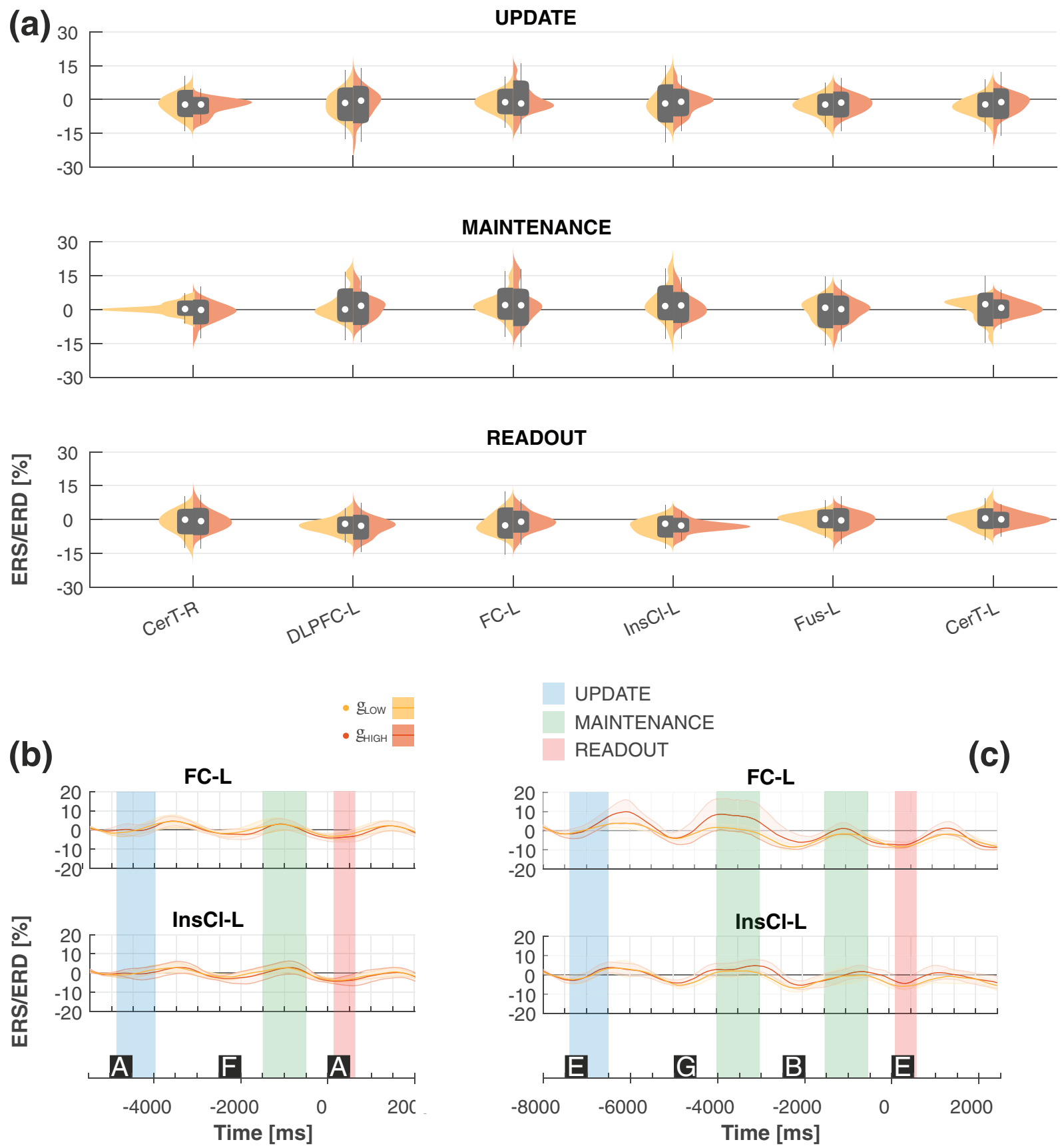

UPDATE

MAINTENANCE

READOUT

(c)

FC-L

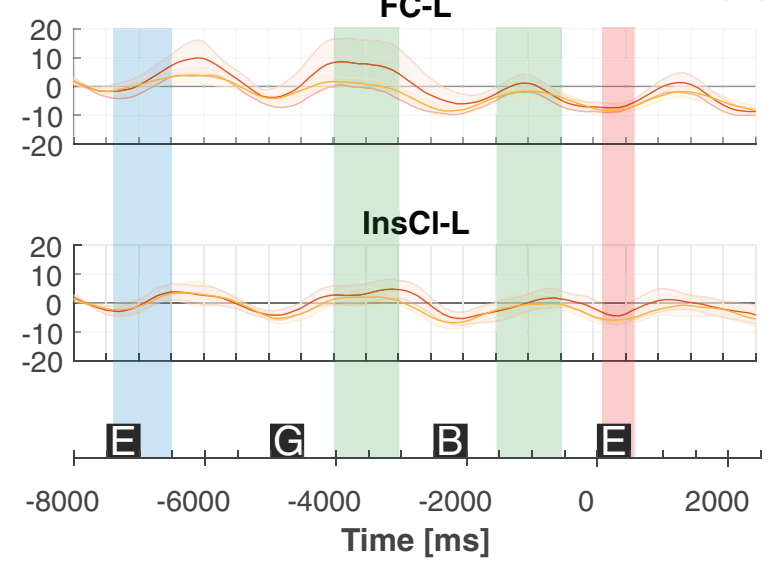

FIGURE 4 Effect of PHASE in the $\gamma$ LOW and $\gamma$ HIGH band. (a) Violin plots of ERS/ERD variation in the $\gamma$ LOW (yellow) and $\gamma$ HIGH (orange) bands during update (top), maintenance (middle) and readout (bottom). Superimposed in grey are boxplots describing the median value (white dot), 25th and 75th percentiles (extremes of the thick grey line), and full data range (extremes of the thin grey line) of the distributions.

(b) Temporal evolution (mean - thick lines, standard deviation - shaded areas) of band power in the $\gamma \mathrm{LOW}$ (yellow) and $\gamma \mathrm{HIGH}$ (orange) during the 2-back task for FC-L and InsCl-L). (c) Temporal evolution of band power in the $\gamma \mathrm{LOW}$ (yellow) and $\gamma \mathrm{HIGH}$ (orange) during the 3-back task for FC-L and InsCl-L

left cortex (fusiform) and left cerebellum depending on the response (ERS in the TP trials and ERD in the TN trials). Noteworthy, only for $\gamma_{\text {LOW }}$ band we observed that some of the modulations in the update and readout of TP trials were stronger for the 3-back than the 2-back task, suggesting that the cognitive load is playing a role in its modulation.

\section{1 $\quad$ EEG oscillations in relation to working memory: The update phase}

Several recent studies highlighted the role played by $\theta$ oscillations in working memory. Particularly, activity in this band has been related to increases in the amount of information to be retained, with $\theta$ 


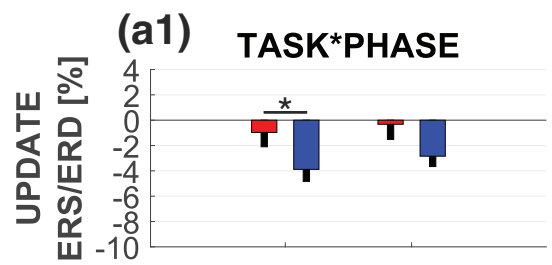

(b1)
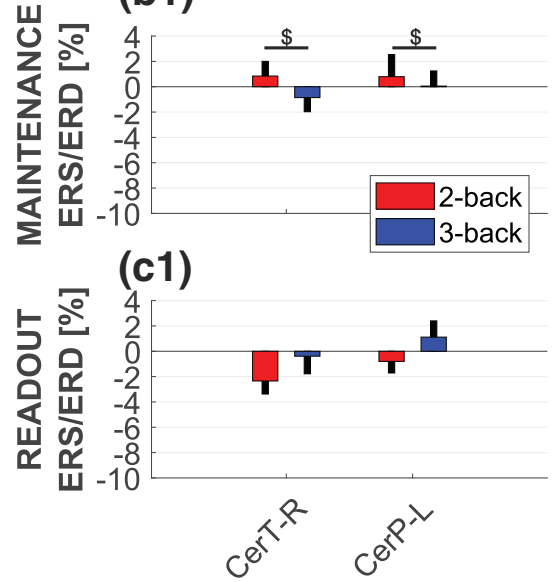

(a2)

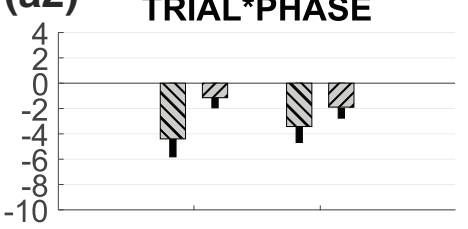

(b2)

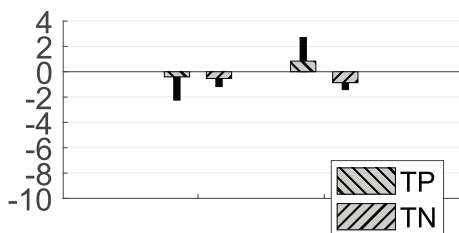

(c2)

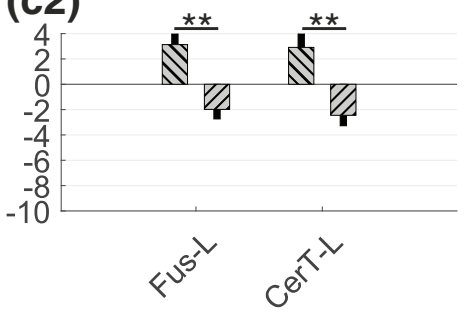

(a3) TASK*TRIAL*PHASE

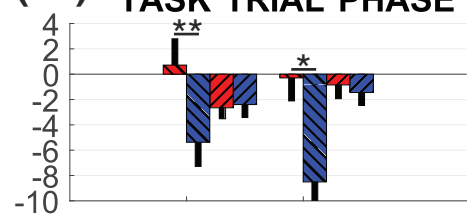

(b3)

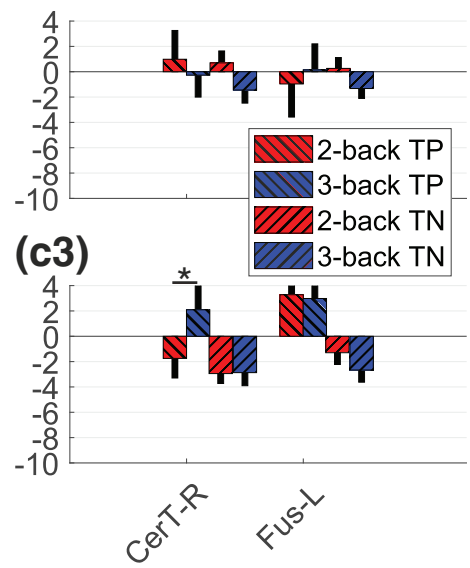

FIGURE 5 Interactions of main effects in the $\gamma$ LOW band. Bar plots of ERS/ERD variation in the $\gamma$ LOW band. Thick bars represent mean across, black thin bars represent standard deviation. Left panels $(a 1, b 1, c 1)$ are referred to TASK*PHASE interaction, central panels (a2, b2, c2) to TRAIL*PHASE interaction, and right panels $(a 3, b 3, c 3)$ to TRIAL*TASK*PHASE interaction. Top panels (a1, a2, a3) represent ERS/ERD during update, central panels (b1, b2, b3) during maintenance and bottom panels (c1, c2, c3) during readout. Level of significance is reported: ${ }^{* *} p<0.01$; ${ }^{*} \mathrm{p}<0.05 ;$ trend

modulations localized to frontal (Gevins, Smith, McEvoy, \& Yu, 1997; Jensen \& Tesche, 2002), hippocampal (Tesche \& Karhu, 2000), and parietal (Sarnthein, Petsche, Rappelsberger, Shaw, \& von Stein, 1998) regions. One of the core functions attributed to $\theta$ band oscillations in the hippocampal system is the temporal integration of cell assemblies (Buzsaki \& Moser, 2013). Although first demonstrated for the tracking of spatial positions, the same mechanism may also support the representation and consolidation of sequentially organized memory traces (Lisman \& Jensen, 2013).

Our findings revealed synchronization in the $\theta$ band in all the areas belonging to WM network following stimulus presentation, consistent with long-range coordination of neuronal activity within WM network. Indeed, neural oscillations are thought to play a central role in coordinating neural activity both in local networks (Gray, Konig, Engel, \& Singer, 1989; Womelsdorf et al., 2007) and over longer distances (von Stein \& Sarnthein, 2000). Particularly, $\delta$ and $\theta$ oscillatory regimens are characterized by long-range interactions (von Stein \& Sarnthein, 2000) requiring communication among several different areas. Simultaneously with an increase of $\theta$ oscillations, we also found a decrease of $\beta$ oscillations at stimulus presentation in parietal and frontal areas of both hemispheres. In a recent study adopting magnetoencephalography (MEG) $\theta$ and $\beta / \gamma$ activity were assessed during the n-back and the Sternberg tasks (Brookes et al., 2011). Similarly, to our results, the authors found increased frontline $\theta$ power together with decreased power in the $\beta / \gamma$ on task initiation. These oscillatory power decreases were most prominent in the $20-40 \mathrm{~Hz}$ frequency band, even if modulation could be observed up to $80 \mathrm{~Hz}$, implying a broadband response (Brookes et al., 2011).

Our findings are also consistent with the literature suggesting that $\theta$ and $\beta / \gamma$ oscillations are linked. While amplitude modulation $\theta$ and $\beta$ bands oscillations were seen in almost all areas of WM network, modulation in $\gamma$ band oscillations were detectable specifically in the clusters containing frontal cortex and insula/claustrum of the left hemisphere.

Noteworthy, recordings of local field potentials with intracranial electrode arrays in animal models (Lundqvist et al., 2016; Lundqvist, Herman, Warden, Brincat, \& Miller, 2018) and in epilepsy patients implanted with intracranial subdural, depth, or subdural and depth electrode arrays in multiple cortical and subcortical brain regions to treat refractory seizures (Kucewicz et al., 2018) showed a role of $\gamma$ band oscillations during update of novel information in a working memory task. Brief $\gamma$ bursts were temporally and spatially linked with the expression of sensory information in spiking during encoding in monkeys (Lundqvist et al., 2016; Lundqvist et al., 2018). High $\gamma$ activities were recorded in response to word encoding in distinct focal areas of the temporal cortex (Kucewicz et al., 2018), possibly indicating local processing of neuronal assemblies (Crone et al., 2006; Lachaux et al., 2012). The physiological source of $\gamma$ activities-local field oscillations or firing of neuronal assemblies-and their role in cognitive function are still actively debated (Crone et al., 2006; Kucewicz et al., 2017; Kucewicz et al., 2018). In this regard, complex processing might not necessarily be associated with highly synchro- 
nized areas, but with different parallel computations and this would then result in stronger but less synchronized activity in the gamma band, resulting in lower gamma power on the scalp EEG, as observed here. However, this is only a possible explanation and future studies may help in better understanding the role of $\gamma$ oscillations in WM update.

\section{2 | EEG oscillations in relation to working memory: The maintenance phase}

Our results were consistent also in the subsequent phase of WM process; that is, the maintenance, with increased $\gamma$ activity in the same areas in which $\gamma$ activity was modulated in the update. In addition to $\gamma_{\text {Low }}$ synchronization in the frontal cortex and insula/claustrum of the left hemisphere, in the maintenance phase we observed increased $\beta$ oscillation (ERS) for most of the selected areas and, accordingly to what observed in the update phase, decreased $\theta$ activity, specifically in the posterior areas.

In human MEG and EEG recordings, the maintenance of visual information in WM is associated with increased $\beta$ and $\gamma$ frequency band amplitudes (Haenschel et al., 2009; Jokisch \& Jensen, 2007; Osipova et al., 2006; Palva, Kulashekhar, Hamalainen, \& Palva, 2011; Tallon-Baudry, Bertrand, Peronnet, \& Pernier, 1998). Related to $\beta$ oscillations, although $\beta$ has been widely studied for movement, it has also been suggested a role in cognitive functions such as WM (Lundqvist et al., 2016; Lundqvist et al., 2018; Lundqvist, Herman, \& Lansner, 2011). Recent studies recorded prefrontal activity in monkeys performing a delayed match-to-sample task, in which several objects had to be encoded, maintained, and tested sequentially over several seconds (Lundqvist et al., 2016). During encoding, brief $\gamma$ bursts were associated with spiking activity while $\beta$ bursts were reduced. Then, in the following delay period, moderate increase of $\beta$ was observed except at the very end, when information was needed again. At that point, $\beta$ was reduced and $\gamma$ increased. The authors speculated that the intermediate elevation of $\beta$ during the delay period relative to the low levels seen at encoding and readout might serve to protect the current working memory contents from interference. Indeed, human studies have shown increases of prefrontal $\beta$ when subjects must filter out distractors (Zavala, Jang, \& Zaghloul, 2017; Zavala, Zaghloul, \& Brown, 2015) or prevent encoding (Hanslmayr, Matuschek, \& Fellner, 2014).

$\gamma$ band oscillations have been suggested to represent a generic mechanism for the representation of individual WM items, irrespective of WM content and format (Roux \& Uhlhaas, 2014). This is because the synchronization of neuronal discharges at $\gamma$ frequencies supports the integration of neurons into cell assemblies in different cortical and subcortical structures (Singer, 2009) and thus could represent an effective representational format for WM information (Roux \& Uhlhaas, 2014). $\gamma$ modulations were observed focally in the prefrontal cortex and insula/claustrum of the left hemisphere. Insula is particularly involved in n-back tasks based on the visual presentation of numbers (Mencarelli et al., 2019), possibly linked to its phonological function (Chee, Soon, Lee, \& Pallier, 2004). Visual WM operations may rely on activation of letter representations in insular cortex, via top-down feedback from neocortical areas including the prefrontal cortex (Moore, Cohen, \& Ranganath, 2006). Thus, top-down input from the prefrontal cortex can additionally promote maintenance of visual images in the face of distraction (Miller, Lundqvist, \& Bastos, 2018; Sakai, Rowe, \& Passingham, 2002). Following this model, ERS in $\gamma$ band in these areas may suggest critical role of this network in visual WM maintenance.

\section{3 | EEG oscillations in relation to working memory: The readout phase}

The temporal window for the readout started together with the update, $100 \mathrm{~ms}$ after letter presentation. Thus the overlap of $\theta / \beta$ oscillations in the readout (i.e.,: increased $\theta$ oscillations and reduced $\beta$ oscillations) with respect to update may be suggestive of the overlap of cognitive processes. Indeed, in n-back task, it is difficult to disentangle between update and readout, since every new stimulus has to be encoded and simultaneously compared with the 2 or 3 stimuli preceding it, in order to be recognized and to trigger the correct response.

However, in addition to $\theta / \beta$ modulation, in the readout we also observed modulation in the $\gamma_{\text {Low }}$ band activity depending on readout process. Indeed, modulation of $\gamma_{\text {LOW }}$ activity differed when decision was to press the button (TP) or not (TN). $\gamma$ oscillations increased in the left fusiform cortex and left cerebellum when subjects had to decide that the probe letter was equal to the stimulus (TP trials) whereas $\gamma$ oscillations decreased in the same areas when subjects had to decide that the probe letter differed from the stimulus (TN trials). In TP trials, increased $\gamma$ oscillations in the readout phase are consistent with recent evidence coming from animal studies with local field potential recordings (Lundqvist et al., 2011; Lundqvist et al., 2016; Lundqvist et al., 2018), suggesting a role for $\gamma$ oscillations when working memory needs to be read out. We also generalize this phenomenon to a process of readout instrumental to inform motor behavior (like a button press). The left fusiform gyrus has been connected with visual word processing (Cohen et al., 2002; Dehaene \& Cohen, 2011; Price \& Devlin, 2011; Wandell, 2011) and represents both phonological information in addition to orthographic information (Zhao et al., 2017). Cerebellar engagement in working memory tasks is reliably reported across multiple studies (Schmahmann \& Pandya, 1997; Strick et al., 2009). Particularly, connections with association cortices (including the prefrontal cortex) are mainly located within posterior cerebellar lobules (including cerebellum tonsil and pyramis), which provide the anatomic substrate for cerebellar involvement in cognition. Taken together, we can suppose a network based on letter recognition, attention based motion processing and selection of WM information for action preparation, specifically active when the response to be selected is a motor output (and not to suppress the motor output, as it happens in the TN trials). 


\subsection{Limitations and perspectives}

One limitation of this work is that no individual volume conductors were constructed for each subject. Although this is a minor drawback for a study focusing on healthy participants, future work should make use of individual head models, especially if neurologic population is involved. However, it is worth mentioning that a recent study found that the use of less precise head models has a relatively smaller impact on EEG source reconstruction than coverage of EEG electrodes over the scalp (Liu et al., 2015).

Another limitation regards the analysis of the correctly performed trials only. Indeed, for healthy participants the 2- and 3-back tasks can be easily performed, generally reaching high performance scores. However, this analysis is only limited to WM mechanisms underlying the processing of correctly encoded information. It will be of interest in future studies to compare between correct and incorrect trials in those populations that are impaired in working memory functions. This will allow to validate the functional significance of the observed oscillations and to better understand the pathophysiological mechanisms underpinning WM impairment.

In the field of noninvasive brain stimulation therapy, EEG-driven tACS is a tool that already showed promising results for restoring cognitive impairments in neurologic patients. Specifically, $\theta-\gamma$ rhythms tACS seems to be able to improve WM performance (Alekseichuk, Turi, Amador de Lara, Antal, \& Paulus, 2016; Reinhart \& Nguyen, 2019). Our results confirm the crucial implication of these frequency bands, and bring up new information regarding most engaged areas and precise timing of their activation in relation to the task. In the context of WM potentiation or restoration, this information may be exploited for setting tACS parameters.

Starting from our findings, future studies should address the role of frequency coupling for coordinating the information flow across the network activated by the different phases of WM.

\section{5 | CONCLUSIONS}

Overall, our study demonstrated specific spectral signatures based on hdEEG associated with updating of memory information, working memory maintenance, and readout, with relatively high spatial resolution. Considering that $\mathrm{n}$-back task is largely used in clinical settings for both diagnosis and rehabilitation, our findings may support the targeted use of noninvasive neuromodulation techniques to boost the WM process in diseases.

\section{ACKNOWLEDGMENTS}

The authors gracefully acknowledge Martina Putzolu and Marta Carè for assistance during data acquisition.

\section{CONFLICT OF INTEREST}

The authors declare no conflict of interest.

\section{AUTHOR CONTRIBUTIONS}

Marianna Semprini, Gaia Bonassi, Elisa Pelosin, Michela Chiappalone, and Laura Avanzino conceived the study. Marianna Semprini, Gaia Bonassi, and Federico Barban collected the data. Marianna Semprini, Gaia Bonassi, Michela Chiappalone, Dante Mantini, and Laura Avanzino defined the analysis. Marianna Semprini, Gaia Bonassi, Federico Barban, and Riccardo landolo performed the analysis. Marianna Semprini prepared the figures. Marianna Semprini, Gaia Bonassi, and Laura Avanzino wrote the paper. All the authors read and approved the final version of the manuscript.

\section{DATA AVAILABILITY STATEMENT}

We plan to make the EEG data sets available on Mendeley-Data repository (or similar). Currently, data are being used for another publication of co-authors of ours and we cannot share the EEG data at this point.

\section{ORCID}

Marianna Semprini (D) https://orcid.org/0000-0001-5504-0251

Riccardo landolo (iD https://orcid.org/0000-0002-5279-1380

Dante Mantini (D) https://orcid.org/0000-0001-6485-5559

\section{REFERENCES}

Alekseichuk, I., Turi, Z., Amador de Lara, G., Antal, A., \& Paulus, W. (2016). Spatial working memory in humans depends on theta and high gamma synchronization in the prefrontal cortex. Current Biology, 26, 1513-1521.

Antal, A., \& Paulus, W. (2013). Transcranial alternating current stimulation (tACS). Frontiers in Human Neuroscience, 7, 317.

Baddeley, A. (1996). The fractionation of working memory. Proceedings of the National Academy of Sciences of the United States of America, 93, 13468-13472.

Brookes, M. J., Wood, J. R., Stevenson, C. M., Zumer, J. M., White, T. P., Liddle, P. F., \& Morris, P. G. (2011). Changes in brain network activity during working memory tasks: A magnetoencephalography study. Neurolmage, 55, 1804-1815.

Burke, J. F., Zaghloul, K. A., Jacobs, J., Williams, R. B., Sperling, M. R., Sharan, A. D., \& Kahana, M. J. (2013). Synchronous and asynchronous theta and gamma activity during episodic memory formation. The Journal of Neuroscience, 33, 292-304.

Buzsaki, G., \& Moser, E. I. (2013). Memory, navigation and theta rhythm in the hippocampal-entorhinal system. Nature Neuroscience, 16, 130-138.

Chee, M. W., Soon, C. S., Lee, H. L., \& Pallier, C. (2004). Left insula activation: A marker for language attainment in bilinguals. Proceedings of the National Academy of Sciences of the United States of America, 101, 15265-15270.

Cohen, L., Lehericy, S., Chochon, F., Lemer, C., Rivaud, S., \& Dehaene, S. (2002). Language-specific tuning of visual cortex? Functional properties of the visual word form area. Brain, 125, 1054-1069.

Crone, N. E., Sinai, A., \& Korzeniewska, A. (2006). High-frequency gamma oscillations and human brain mapping with electrocorticography. Progress in Brain Research, 159, 275-295.

Dehaene, S., \& Cohen, L. (2011). The unique role of the visual word form area in reading. Trends in Cognitive Sciences, 15, 254-262.

Gevins, A., Smith, M. E., McEvoy, L., \& Yu, D. (1997). High-resolution EEG mapping of cortical activation related to working memory: Effects of task difficulty, type of processing, and practice. Cerebral Cortex, 7, 374-385. 
Gray, C. M., Konig, P., Engel, A. K., \& Singer, W. (1989). Oscillatory responses in cat visual cortex exhibit inter-columnar synchronization which reflects global stimulus properties. Nature, 338, 334-337.

Haenschel, C., Bittner, R. A., Waltz, J., Haertling, F., Wibral, M., Singer, W., ... Rodriguez, E. (2009). Cortical oscillatory activity is critical for working memory as revealed by deficits in early-onset schizophrenia. The Journal of Neuroscience, 29, 9481-9489.

Hanslmayr, S., Matuschek, J., \& Fellner, M. C. (2014). Entrainment of prefrontal beta oscillations induces an endogenous echo and impairs memory formation. Current Biology, 24, 904-909.

Haueisen, J., Bottner, A., Funke, M., Brauer, H., \& Nowak, H. (1997). Effect of boundary element discretization on forward calculation and the inverse problem in electroencephalography and magnetoencephalography. Biomedizinische Technik. Biomedical Engineering, 42, 240-248.

Helfrich, R. F., Schneider, T. R., Rach, S., Trautmann-Lengsfeld, S. A., Engel, A. K., \& Herrmann, C. S. (2014). Entrainment of brain oscillations by transcranial alternating current stimulation. Current Biology, 24, 333-339.

Herrmann, C. S., Rach, S., Neuling, T., \& Struber, D. (2013). Transcranial alternating current stimulation: A review of the underlying mechanisms and modulation of cognitive processes. Frontiers in Human Neuroscience, 7, 279.

Hill, A. T., Rogasch, N. C., Fitzgerald, P. B., \& Hoy, K. E. (2019). Impact of concurrent task performance on transcranial direct current stimulation (tDCS)-induced changes in cortical physiology and working memory. Cortex, 113, 37-57.

Honkanen, R., Rouhinen, S., Wang, S. H., Palva, J. M., \& Palva, S. (2015). Gamma oscillations underlie the maintenance of feature-specific information and the contents of visual working memory. Cerebral Cortex, 25, 3788-3801.

Hoy, K. E., Bailey, N., Arnold, S., Windsor, K., John, J., Daskalakis, Z. J., \& Fitzgerald, P. B. (2015). The effect of gamma-tACS on working memory performance in healthy controls. Brain and Cognition, 101, 51-56.

Hoy, K. E., Bailey, N., Michael, M., Fitzgibbon, B., Rogasch, N. C., Saeki, T., \& Fitzgerald, P. B. (2016). Enhancement of working memory and task-related oscillatory activity following intermittent theta burst stimulation in healthy controls. Cerebral Cortex, 26, 4563-4573.

Hsieh, L. T., \& Ranganath, C. (2014). Frontal midline theta oscillations during working memory maintenance and episodic encoding and retrieval. Neurolmage, 85(Pt 2), 721-729.

Hyvarinen, A., \& Oja, E. (2000). Independent component analysis: Algorithms and applications. Neural Networks, 13, 411-430.

Jensen, O., \& Tesche, C. D. (2002). Frontal theta activity in humans increases with memory load in a working memory task. The European Journal of Neuroscience, 15, 1395-1399.

Jokisch, D., \& Jensen, O. (2007). Modulation of gamma and alpha activity during a working memory task engaging the dorsal or ventral stream. The Journal of Neuroscience, 27, 3244-3251.

Jones, K. T., Johnson, E. L., \& Berryhill, M. E. (2020). Frontoparietal thetagamma interactions track working memory enhancement with training and tDCS. Neurolmage, 211, 116615.

Kirchner, W. K. (1958). Age differences in short-term retention of rapidly changing information. Journal of Experimental Psychology, 55, 352-358.

Kucewicz, M. T., Berry, B. M., Kremen, V., Brinkmann, B. H., Sperling, M. R., Jobst, B. C., ... Worrell, G. A. (2017). Dissecting gamma frequency activity during human memory processing. Brain, 140, 1337-1350.

Kucewicz, M. T., Berry, B. M., Kremen, V., Miller, L. R., Khadjevand, F., Ezzyat, Y., ... Worrell, G. A. (2018). Electrical stimulation modulates high $\gamma$ activity and human memory performance. Eneuro, 5, 0369-0317.

Lachaux, J. P., Axmacher, N., Mormann, F., Halgren, E., \& Crone, N. E. (2012). High-frequency neural activity and human cognition: Past, present and possible future of intracranial EEG research. Progress in Neurobiology, 98, 279-301.

Lisman, J. E., \& Jensen, O. (2013). The theta-gamma neural code. Neuron, 77, 1002-1016.

Liu, Q., Balsters, J. H., Baechinger, M., van der Groen, O., Wenderoth, N., \& Mantini, D. (2015). Estimating a neutral reference for electroencephalographic recordings: The importance of using a high-density montage and a realistic head model. Journal of Neural Engineering, 12, 056012.

Liu, Q., Farahibozorg, S., Porcaro, C., Wenderoth, N., \& Mantini, D. (2017). Detecting large-scale networks in the human brain using high-density electroencephalography. Human Brain Mapping, 38, 4631-4643.

Liu, Q., Ganzetti, M., Wenderoth, N., \& Mantini, D. (2018). Detecting large-scale brain networks using EEG: Impact of electrode density, head modeling and source localization. Frontiers in Neuroinformatics, $12,4$.

Lundqvist, M., Herman, P., \& Lansner, A. (2011). Theta and gamma power increases and alpha/beta power decreases with memory load in an attractor network model. Journal of Cognitive Neuroscience, 23, 3008-3020.

Lundqvist, M., Herman, P., Warden, M. R., Brincat, S. L., \& Miller, E. K. (2018). Gamma and beta bursts during working memory readout suggest roles in its volitional control. Nature Communications, 9, 394.

Lundqvist, M., Rose, J., Herman, P., Brincat, S. L., Buschman, T. J., \& Miller, E. K. (2016). Gamma and Beta bursts underlie working memory. Neuron, 90, 152-164.

Mantini, D., Franciotti, R., Romani, G. L., \& Pizzella, V. (2008). Improving MEG source localizations: An automated method for complete artifact removal based on independent component analysis. Neurolmage, 40, 160-173.

Mencarelli, L., Neri, F., Momi, D., Menardi, A., Rossi, S., Rossi, A., \& Santarnecchi, E. (2019). Stimuli, presentation modality, and loadspecific brain activity patterns during n-back task. Human Brain Mapping, 40, 3810-3831.

Michel, C. M., \& Murray, M. M. (2012). Towards the utilization of EEG as a brain imaging tool. Neurolmage, 61(2), 371-385. https://doi.org/10. 1016/j.neuroimage.2011.12.039.

Miller, E. K., Lundqvist, M., \& Bastos, A. M. (2018). Working memory 2.0. Neuron, 100, 463-475.

Moore, C. D., Cohen, M. X., \& Ranganath, C. (2006). Neural mechanisms of expert skills in visual working memory. The Journal of Neuroscience, 26 , 11187-11196.

Oostenveld, R., \& Praamstra, P. (2001). The five percent electrode system for high-resolution EEG and ERP measurements. Clinical Neurophysiology: Official Journal of the International Federation of Clinical Neurophysiology, 112, 713-719.

Osipova, D., Takashima, A., Oostenveld, R., Fernandez, G., Maris, E., \& Jensen, O. (2006). Theta and gamma oscillations predict encoding and retrieval of declarative memory. The Journal of Neuroscience, 26, 7523-7531.

Palva, S., Kulashekhar, S., Hamalainen, M., \& Palva, J. M. (2011). Localization of cortical phase and amplitude dynamics during visual working memory encoding and retention. The Journal of Neuroscience, 31, 5013-5025.

Pascual-Marqui, R. D., Lehmann, D., Koukkou, M., Kochi, K., Anderer, P., Saletu, B., ... Kinoshita, T. (2011). Assessing interactions in the brain with exact low-resolution electromagnetic tomography. Philosophical Transactions. Series A, Mathematical, Physical, and Engineering Sciences, 369, 3768-3784.

Pfurtscheller, G. (2001). Functional brain imaging based on ERD/ERS. Vision Research, 41, 1257-1260.

Price, C. J., \& Devlin, J. T. (2011). The interactive account of ventral occipitotemporal contributions to reading. Trends in Cognitive Sciences, $15,246-253$ 
Reinhart, R. M. G., \& Nguyen, J. A. (2019). Working memory revived in older adults by synchronizing rhythmic brain circuits. Nature Neuroscience, 22, 820-827.

Roux, F., \& Uhlhaas, P. J. (2014). Working memory and neural oscillations: Alpha-gamma versus theta-gamma codes for distinct WM information? Trends in Cognitive Sciences, 18, 16-25.

Roux, F., Wibral, M., Mohr, H. M., Singer, W., \& Uhlhaas, P. J. (2012). Gamma-band activity in human prefrontal cortex codes for the number of relevant items maintained in working memory. The Journal of Neuroscience, 32, 12411-12420.

Sakai, K., Rowe, J. B., \& Passingham, R. E. (2002). Active maintenance in prefrontal area 46 creates distractor-resistant memory. Nature Neuroscience, 5, 479-484.

Sarnthein, J., Petsche, H., Rappelsberger, P., Shaw, G. L., \& von Stein, A. (1998). Synchronization between prefrontal and posterior association cortex during human working memory. Proceedings of the National Academy of Sciences of the United States of America, 95, 7092-7096.

Schmahmann, J. D., \& Pandya, D. N. (1997). The cerebrocerebellar system. International Review of Neurobiology, 41, 31-60.

Singer, W. (2009). Distributed processing and temporal codes in neuronal networks. Cognitive Neurodynamics, 3, 189-196.

Strick, P. L., Dum, R. P., \& Fiez, J. A. (2009). Cerebellum and nonmotor function. Annual Review of Neuroscience, 32, 413-434.

Tallon-Baudry, C., Bertrand, O., Peronnet, F., \& Pernier, J. (1998). Induced gamma-band activity during the delay of a visual short-term memory task in humans. The Journal of Neuroscience, 18, 4244-4254.

Tesche, C. D., \& Karhu, J. (2000). Theta oscillations index human hippocampal activation during a working memory task. Proceedings of the National Academy of Sciences of the United States of America, 97, 919-924.

Thorpe, S., Fize, D., \& Marlot, C. (1996). Speed of processing in the human visual system. Nature, 381, 520-522.

von Stein, A., \& Sarnthein, J. (2000). Different frequencies for different scales of cortical integration: From local gamma to long range alpha/theta synchronization. International Journal of Psychophysiology, 38, 301-313
Wandell, B. A. (2011). The neurobiological basis of seeing words. Annals of the New York Academy of Sciences, 1224, 63-80.

Womelsdorf, T., Schoffelen, J. M., Oostenveld, R., Singer, W., Desimone, R., Engel, A. K., \& Fries, P. (2007). Modulation of neuronal interactions through neuronal synchronization. Science, 316, 1609-1612.

Yao, D. (2001). A method to standardize a reference of scalp EEG recordings to a point at infinity. Physiological Measurement, 22, 693-711.

Yao, D., Wang, L., Oostenveld, R., Nielsen, K. D., Arendt-Nielsen, L., \& Chen, A. C. (2005). A comparative study of different references for EEG spectral mapping: The issue of the neutral reference and the use of the infinity reference. Physiological Measurement, 26, 173-184.

Zavala, B., Zaghloul, K., \& Brown, P. (2015). The subthalamic nucleus, oscillations, and conflict. Movement Disorders, 30, 328-338.

Zavala, B. A., Jang, A. I., \& Zaghloul, K. A. (2017). Human subthalamic nucleus activity during non-motor decision making. eLife, 6.

Zhao, L., Chen, C., Shao, L., Wang, Y., Xiao, X., Chen, C., ... Xue, G. (2017). Orthographic and phonological representations in the fusiform cortex. Cerebral Cortex, 27, 5197-5210.

Zhao, M., Marino, M., Samogin, J., Swinnen, S. P., \& Mantini, D. (2019). Hand, foot and lip representations in primary sensorimotor cortex: A high-density electroencephalography study. Scientific Reports, 9, 19464.

\section{SUPPORTING INFORMATION}

Additional supporting information may be found online in the Supporting Information section at the end of this article.

How to cite this article: Semprini M, Bonassi G, Barban F, et al. Modulation of neural oscillations during working memory update, maintenance, and readout: An hdEEG study. Hum Brain Mapp. 2021;42:1153-1166. https://doi.org/10.1002/ hbm.25283 